\title{
ANALYSIS OF DIFFERENT POLARIMETRIC DECOMPOSITION TECHNIQUES USING COMPACT POLARIMETRIC NISAR DATA FOR AHMEDABAD, INDIA
}

\author{
A. Vyas $^{1^{*}}$, V. Nizalapur ${ }^{1 *}$, P. Chhasia, D. Rawal ${ }^{1}$ \\ G. Jain ${ }^{2}$, A. Das ${ }^{2}$ \\ ${ }^{1}$ Center for Applied Geomatics, CRDF, CEPT University, Ahmedabad, Gujarat, India \\ ${ }^{2}$ Space Applications Centre, ISRO, Ahmedabad, Gujarat, India. \\ Email: n.vyjayanthi@gmail.com; anjanavyas@yahoo.com
}

\section{Commission III, WG II}

KEY WORDS: Compact polarimetry, Polarimetric Decomposition, m-Alpha, m-Chi, m-Delta, NISAR

\begin{abstract}
:
The present study addresses the potential of airborne NASA - ISRO Synthetic Aperture Radar (NISAR) compact polarimetric (CP) data to discriminate the land cover classes emphasizing the urban area for parts of Ahmedabad city, India. This has been carried out by generating m-Delta, $\mathrm{m}$-Chi and $\mathrm{m}$-Alpha polarimetric decompositions using Compact Polarimetric L band NISAR data. In Hybrid Polarimetric data, both m-delta and $\mathrm{m}$-chi decompositions have almost the same formulations, indicating that delta and chi play the same roles as indicators of single-bounce and double-bounce scattering. However, M-delta seem preferable over M-chi as stoke parameter delta is highly susceptible towards orientation. It is also observed that building orientation and density has effect on scattering pattern. This is attributed to the target orientation which is parallel to the look direction of the sensor. Supervised classification of m-Delta decomposition was carried out and over all accuracy of $81.1 \%$ was observed in the study.
\end{abstract}

\section{INTRODUCTION}

SAR data are the best suited for monitoring various land covers targets as well as target parameters retrieval due to its allweather capability and unique sensitivity to all the geometrical and structural properties of the target.

A hybrid version that transmits a circularly polarized wave ( $R$ or $\mathrm{L}$ ) and receives $\mathrm{H}$ and $\mathrm{V}$ is known as compact-polarimetry. Compact-pol combines the properties of dual-pol, e.g., discrimination between oriented and random surfaces, while better balancing the power between the receive channels

Compact-polarimetric orbital systems viz, NASA's Lunar Reconnaissance Orbiter (LRO) Chandrayan-1, (Saran et al., 2011; Raney et al., 2011) RISAT-1 (Uppala et al., 2015; Xie et al, 2015; Sivasankar et al., 2019) were very useful to understand and analyse scattering mechanisms of different targets using stokes parameters, stokes child parameters and polarimetric decompositions.

Stokes parameters are represented by four real numbers $\left(S_{0}, S_{1}\right.$, $\mathrm{S}_{2}, \mathrm{~S}_{3}$ ), and are suitable to describe the received polarization state and scattering properties of targets. The parameters derived from Stokes parameters are known as Stokes child parameters (Raney et al., 2006). They are degree of polarization $(\mathrm{m})$, relative phase $(\delta)$, ellipticity parameter $(\chi)$, polarization angle $(\alpha)$ and circular polarization ratio $(\mu)$. The equations to derive stoke child parameters and polarimetric decompositions are given by Raney et al., 2007 and Raney et al., 2012.

Supervised classification methods for the polarimetric SAR data can be divided into statistical and neural network approaches. Neural network techniques have also been applied using the complete polarimetric information as input, and iterative training was normally necessary. Neural network classification shows better result with respect to other classification methods, edges of building are better preserved in this classification.

Due to the high variability of urban landscape, its complex combinations of natural and man-made targets, and object forms and sizes, the scattering mechanisms are much more complex in built-up area when compared with natural targets. The three types of scattering mechanisms possible in urban area are i) even, i) volume and iii) odd bounce. Target decomposition of polarimetric SAR data is essential to understand the predominant scattering type.

Several studies (Charbonneau et al., 2010) studied the applications of compact polarimetric data for land cover targets in Indian context (Turkar et al., 2013; Sivasankar et al., 2015; Jayasri et al., 2018) but very less studies focussed on the analysis within the built-up area.

The main objective in the present study is to understand and analyse scattering mechanism of urban built forms and other land features using stokes child parameters and decomposition techniques.

Building orientation with respect to the radar look direction has a critical influence on the interpretation of polarimetric synthetic aperture radar (PolSAR) data in urban areas. This paper also attempted to study the effect of orientation of the target on scattering mechanisms in different decompositions and variation of scattering with respect to height (Rise) of the building and density (sparse or dense).

\section{DATA DESCRIPTION AND STUDY AREA}

*corresponding author 
Airborne NASA - ISRO Synthetic Aperture Radar (NISAR) compact polarimetric (CP) data was acquired on 16th June, 2017 with central latitude of $23.04 \mathrm{~N}$ and longitude of $72.46 \mathrm{E}$ in $\mathrm{L}(1250 \mathrm{~m} \mathrm{~Hz})$ wavelength band. The sensor orientation is left and the look angle is $39.1^{\circ}$. The near and far angles are $18^{\circ}$ and $48.7^{\circ}$ respectively. Pizel spacing is $1.8 \mathrm{~m} \mathrm{X} 2 \mathrm{~m}$ in range and azimuth direction.

The study area is Ahmedabad city of Gujarat state Ahmedabad is an urban, densely populated industrialized largest city in the central part of the Gujarat state in Western India. Only one image of NISAR was available in lower North West region of Ahmedabad viz., Bopal region for analysis.

The land use/land cover (LULC) types in the study area were broadly divided into Urban (High Rise and Low Rise), Water (Rivers, Lakes, Canals), Vegetation (Crop Land, Scrub, Parks), and Open Land. The land cover in study area is diverse; certain land cover types exhibit similar scattering mechanisms, which make difficult to identify those features.

\section{METHODOLOGY}

Methodology followed in the present study is given in Fig 1 . First, Stokes parameters $\left(S_{1}, S_{2}, S_{3}\right.$ and $\left.S_{4}\right)$ are derived using single look complex (SLC) NISAR data of L-band. From Stokes Parameters, Stokes Child Parameters viz., degree of polarization $(\mathrm{m})$, relative phase $(\delta)$, ellipticity parameter $(\chi)$, polarization angle $(\alpha)$ and circular polarization ratio $(\mu)$ are derived. Speckle suppression is carried out using polarimetric Refined Lee filter of window size 5X5. Stokes and its child parameters are derived using the equations given in literature They are carried out using band math function in ENVI software. All the derived parameters are analysed for different land cover classes. Three polarimetric decomposition techniques namely m-delta, $\mathrm{m}$-alpha and $\mathrm{m}$-chi are carried out to understand the scattering mechanisms of different land cover classes.

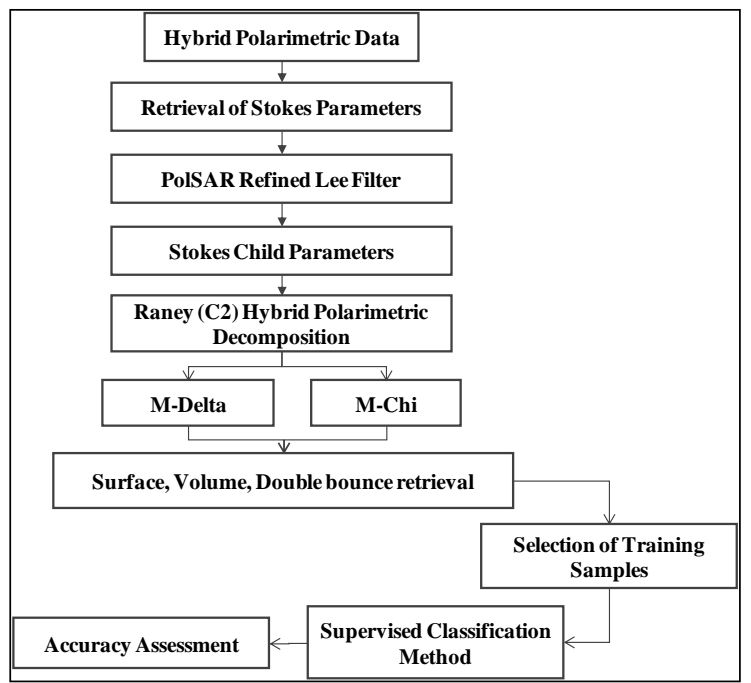

Figure 1: Methodology flowchart - Polarimetric decompositions - Classification

Also, Region of interests (ROIs) for different land cover classes are drawn to anlayse the variation of $\mathrm{m}$, chi, delta and alpha parameters. The variation of scattering mechanism/power is studied for different orientation of the buildings in the study area.
Comparative analysis of all the decompositions is carried out and m-delta decomposition which showed better discrimination is further used for supervised classification using Neural Network method. Accuracy assessment is carried out using high resolution Google Earth imageries.

\section{RESULTS AND DISCUSSIONS}

\subsection{Stokes child parameters}

The histograms of stokes child parameters are analysed for different land cover classes in the study area and are explained in detail below Stokes parameters are given as S1, S2,S3, S4 and readers can refer Raney et al, 2012 for detailed explanation.

4.1.1 Circular Polarization Ratio ( $\mu$ ): CPR is an index that shows how the received energy is allocated between different polarization states during the interaction with the target. It is also one of the criterions for scattering dominance It is given by the below equation:

$$
\text { Circular polarization Ratio, } \mu=\underline{\text { (S }}
$$

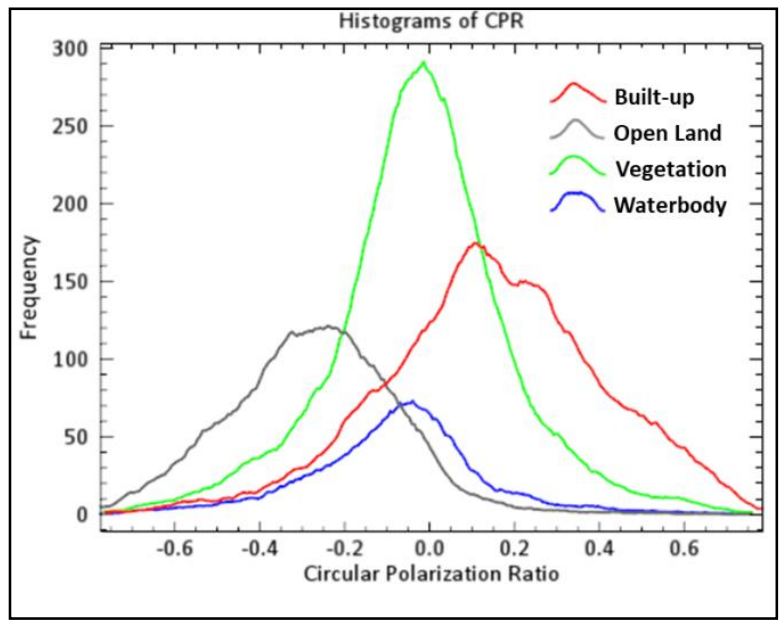

Figure 2: Histogram of CPR in different Land cover classes

From the fig 2, the variation of CPR for different land cover classes is studied.

- Built-up shows high degree of circularity because of double bounce scattering.

- Vegetation and water also shows high degree of circularity due to more contribution of volume scattering and odd bounce scattering from water.

4.1.2 Degree of Polarization (DOP), $\mathrm{m}$ : The degree of polarization, $\mathrm{m}$ is given by the formula

- The higher polarized signal is observed in vegetation followed by built-up areas.

- Broad structure of vegetation is like a vertically oriented dipole and buildings have well defined structures and hence can be viewed as dihedral corner reflectors. 
- Due to random nature of polarized or un-polarized return signal from water, open land shows low degree of polarization.

- The value of degree of polarization lies in between 0 to 1 $(0 \leq \mathrm{m} \leq 1)$ where $\mathrm{m}=1$ represents the totally polarized wave.

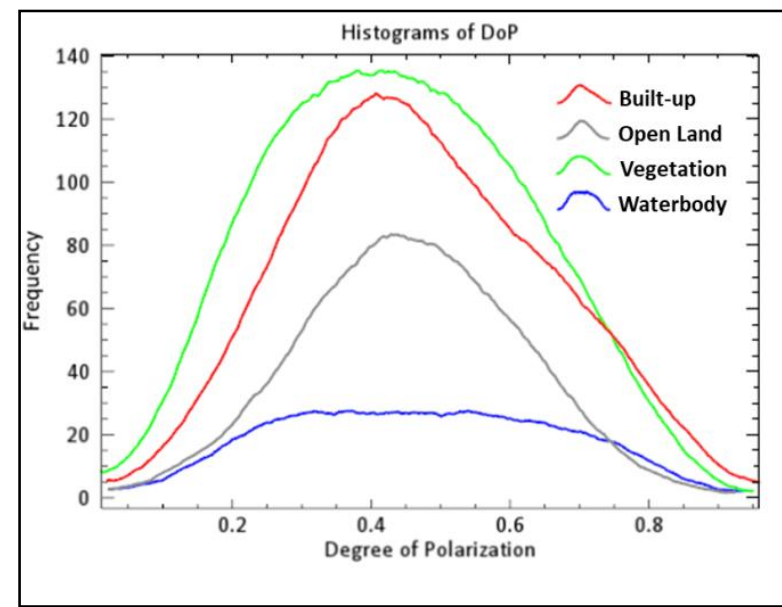

Figure 3: Histogram of DoP in different Land cover classes

4.1.3 Relative Phase ( $\delta$ ): The relative phase angle between the RH and RV polarizations in CP SAR data includes the phase information of the backscatter of the targets. It is given by the formula

$$
\text { Relative phase, } \delta=a \tan \left(\frac{S_{3}}{s_{2}}\right)
$$

- For built-up mean value of relative phase are negative which indicates that double bounce is dominant.

- All other classes have positive phase mean value because of less contribution in double bounce.

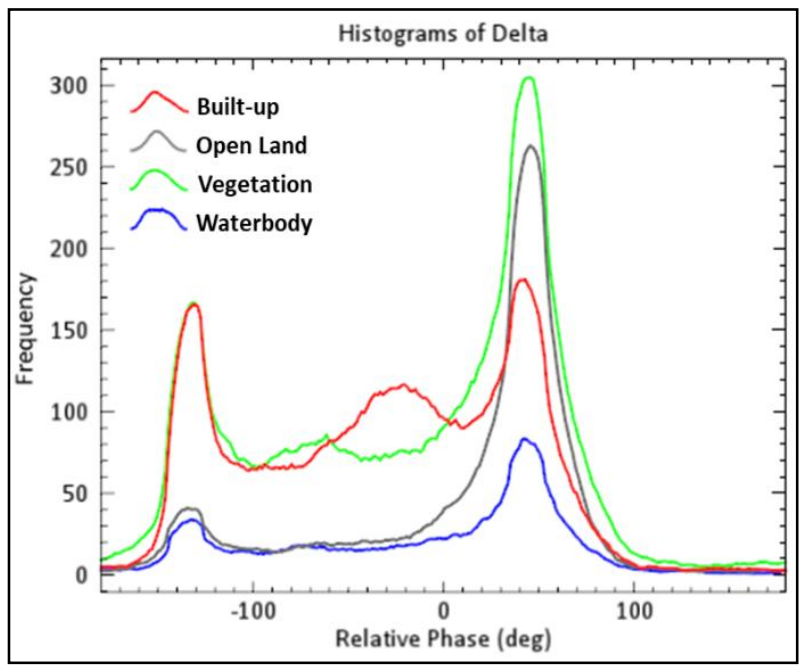

Figure 4: Histogram of Relative phase, $\delta$ in different Land cover classes

\subsubsection{Polarization angle ( $\alpha)$ : Polarization angle is} calculated using Stokes parameters as

$$
\text { Alpha, } \alpha=\frac{1}{2} \tan ^{-1}\left(\frac{\sqrt{\mathrm{S}_{1}^{2}+\mathrm{S}_{2}^{2}}}{\mathrm{~S}_{3}}\right) \text {. }
$$

This $\alpha$ parameter can only be estimated from Hybrid PolSAR data when there is dominant scattering from built-up.

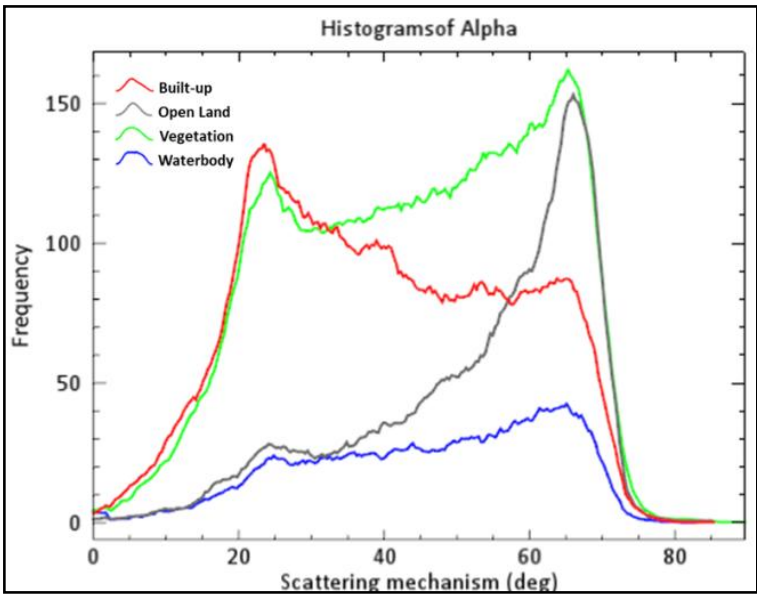

Figure 5: Histogram of Alpha in different Land cover classes

On the whole, it is observed that vegetation, built-up and water show high degree of circularity $(\mu)$ due to more contribution of volume scattering, double bounce scattering and odd bounce scattering respectively. The higher degree of polarization $(\mathrm{m})$ is observed in vegetation followed by built-up areas. The relative phase $(\delta)$ mean value for built-up negative which indicates that double bounce is dominant.

\subsection{Polarimetric decompositions}

Three decomposition techniques $\mathrm{m}$-delta, $\mathrm{m}$-alpha and $\mathrm{m}$-chi are carried out. These target decomposition theorems identify the different scattering mechanisms like surface scattering, double bounce scattering and multiple scattering in relation to the targets that are of user's interest (urban, agriculture etc.) The equations of the three decompositions are also given in Jayasri et al., 2018.

4.2.1 M-delta (m- $\boldsymbol{\delta})$ decomposition: The m-delta decomposition comprises of degree of polarization $(\mathrm{m})$ and relative phase $(\delta)$. Raney et al.2007, introduced the practical applicability of $(\mathrm{m}-\delta)$ decomposition. Degree of polarization, $\mathrm{m}$ is indicating polarized and diffuse scattering. Relative phase, $\delta$ (here phase between RH and RV) is indicating double bounce scattering. $\mathrm{m}$ and $\delta$ are able to characterize the polarization state of electromagnetic wave and can be expressed with the help of Stokes parameters.

In this decomposition technique, $\mathrm{m}$ is the sensitive indicator of volume scattering and $\delta$ is the sensitive indicator of even bounce against odd bounce scattering.

4.2.2 M-chi (m- $\chi$ ) decomposition: Raney et al.,2010 applied the $(\mathrm{m}-\chi)$ decomposition for hybrid polarimetric data. The $(\mathrm{m}-\chi)$ decomposition consists of degree of polarization (m) and ellipticity parameter $(\chi)$. This decomposition model is using parameters $m$ and $\chi$ which are derived from Stokes parameters. The DoP is indicating diffuse scattering while $\chi$ is an indicator of even versus odd scattering. The value of DoP lies in between 0 to $1(0 \leq \mathrm{m} \leq 1)$ while chi lies in between $-45^{\circ}$ to $+45^{\circ}\left(-45^{\circ}\right.$ $\left.\leq \delta \leq+45^{\circ}\right)$. The $(\chi)$ enters in this decomposition modelling in the form of degree of circularity.

4.2.3 M-Alpha (m- $\alpha$ ) decomposition: The m-alpha decomposition comprises of DoP (m) and polarization angle 
$(\alpha)$. This decomposition method is based on Eigenvector analysis of Hybrid PolSAR data and similar to $\mathrm{H} / \alpha$ decomposition of fully polarimetric data. This $\alpha$ parameter can only be estimated from Hybrid PolSAR data when there is dominant scattering from urban and agriculture fields.

4.2.4 Comparative analysis of different features in $\mathbf{m}-\boldsymbol{\chi}$, $\mathbf{m}-\boldsymbol{\delta}, \mathbf{m}-\boldsymbol{\alpha}$ decompositions: Figure 6 shows the different classes within the built-up area viz., Buildings with different orientations and buildings with rise and density in m-delta decomposed image. It can be observed that yellow colour corresponds to the double bounce from built-up areas i.e., buildings; green colour corresponds to the volume scattering from vegetation. The blue colour corresponds to the surface scattering from crop fields, bare soil and dark blue corresponds to water bodies.

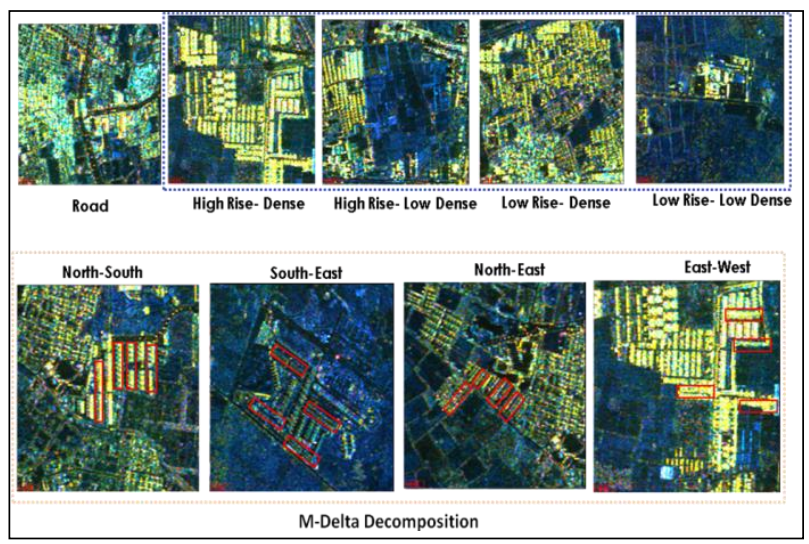

Figure 6: Different classes emphasizing in built-up area as shown in $\mathrm{m}$-delta decomposed image

Built-up areas are easily distinguishable due to their high backscatter and appear bright in the image. Vegetation undergoes volume scattering and hence have medium backscatter. Agricultural boundaries are also distinguished from the image. Water body which undergoes specular reflection has low back scatter and appears dark in the image.

Figure 7 shows the scattering power of different land use classes in m-delta, m-chi and m-alpha decompositions. In mdelta decomposition, even/double bounce scattering is dominant in built-up area followed by volume scattering. The built-up area consists of buildings, houses and concrete structures. The backscatter from this class is dominantly volume scattering of $45 \%$, but even/double-bounce component of about $38 \%$ is also present. Dominant scattering mechanism for vegetation is volume scattering followed by double bounce scattering. The water class has a dominant volume scatter component of about $45 \%$ and odd scatter component of $34 \%$ due to surface roughness. The scattering observed over open land is primarily of volume type of about $47 \%$ and even component of $12 \%$. The return from the crop area is predominantly even bounce with $45 \%$ of the scattered power. However, it has a significant odd component of about $28 \%$ and volume component of $27 \%$. Hence, we can say that the scattering mechanism of each class is distinct in m-delta decomposition.

In m-chi and m-alpha decompositions, there is not much variation of scattering powers of different land cover classes as shown in the figure 7.

Visual analysis showed that m-delta has better image visualization than $\mathrm{m}$-chi due to less noise in the image. Results indicate that delta and chi play the same roles as indicators of single-bounce and double-bounce scattering. However, m-delta seems preferable over $\mathrm{m}$-chi as stoke parameter delta is highly susceptible towards orientation of the target.

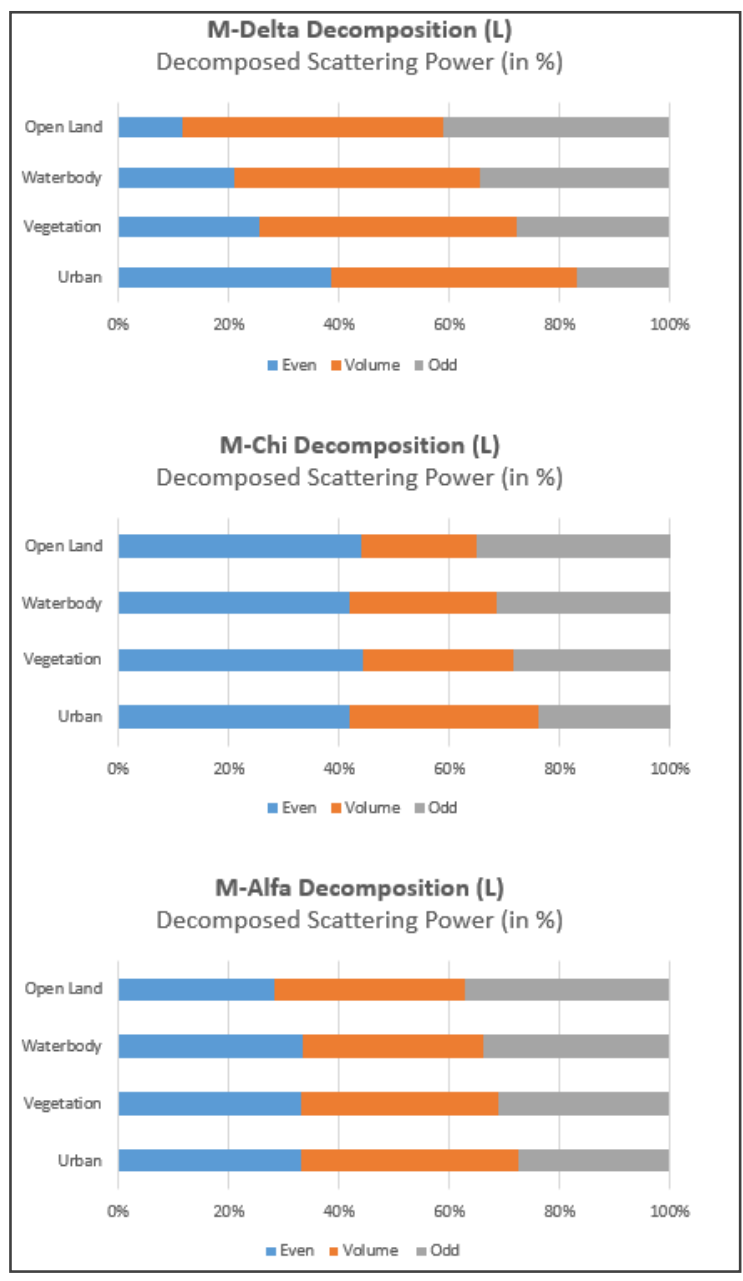

Figure 7: Percentage scattering power (Even, Volume, Odd) for different land use classes

4.2.5 Effect of building orientation on scattering power: Many researchers have found that there exist two types of difficulties in detection or identification in built-up area. One, oriented buildings with respect to radar line of sight which gives volume scattering when compared to double-bounce scattering from dihedral structures of orthogonal buildings. And, second, plantation within the built-up area which exhibits similar scattering (volume) of oriented buildings makes it difficult to distinguish them. It is understood that building orientation and density has effect on scattering pattern.

As shown in Figure 6, several Regions of interests (ROIs) are selected to analyse the variation of scattering powers in different polarimetric decomposition images with respect to rise and orientation of the buildings. Graphical representation is carried out and the results are tabulated to understand the scattering mechanisms.

It is also observed that buildings not aligned towards radar look direction undergoes volume scattering whereas buildings that are orthogonal to Radar line of Sight shows double-bounce scattering mechanism. 
From the Table 1, we can conclude that high even/double bounce scattering power is observed for North South orientation buildings followed by East-West orientation buildings.

\begin{tabular}{|l|l|l|l|l|l|l|}
\hline \multicolumn{4}{|c}{ M- Chi } & \multicolumn{3}{c|}{ M-Delta } \\
\multicolumn{2}{|c|}{ Orientation Even } & \multicolumn{1}{c|}{ Volume } & Odd & Even & \multicolumn{1}{c|}{ Volume } & Odd \\
\hline EW & $39.74 \%$ & $45.32 \%$ & $14.94 \%$ & $42.78 \%$ & $35.21 \%$ & $22.01 \%$ \\
\hline NE & $33.39 \%$ & $46.29 \%$ & $20.32 \%$ & $44.04 \%$ & $31.47 \%$ & $24.49 \%$ \\
\hline NS & $44.07 \%$ & $40.65 \%$ & $15.28 \%$ & $41.39 \%$ & $35.37 \%$ & $23.24 \%$ \\
\hline SE & $25.80 \%$ & $45.67 \%$ & $28.53 \%$ & $43.48 \%$ & $28.04 \%$ & $28.48 \%$ \\
\hline
\end{tabular}

Table 1: Percentage of scattering powers of different orientation of buildings

This is attributed to the target orientation which is parallel to the look direction of the sensor. Volume scattering power is dominant oriented buildings.

In our study area, North-South oriented buildings show relatively high-volume scattering in $\mathrm{m}$-delta and $\mathrm{m}$-chi decomposition, however, has the lowest odd scattering in all decompositions. It is noticeable that in m-chi decomposition. South-East oriented buildings show low scattering values in all decomposition. East-West oriented buildings follow similar trend of North-South oriented buildings in all decomposition.

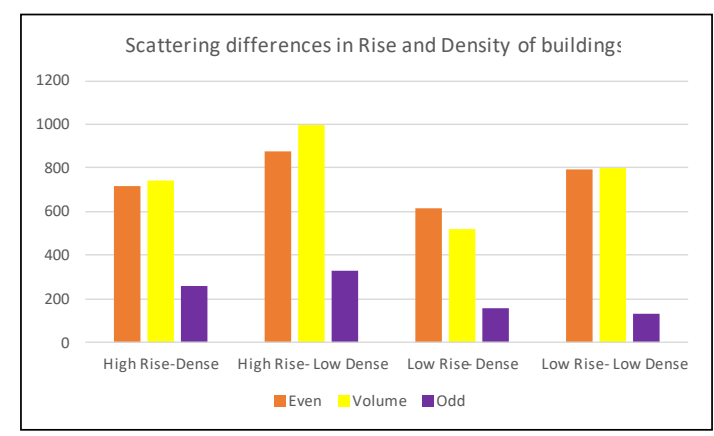

Figure 8: Variation of scattering power with respect to rise and density of buildings

From Figure 8, High rise low dense buildings are showing high scattering power in even and volume scattering mechanisms. This may be because the open land between the two buildings may contribute for double bounce effect and also plantation/vegetation within the built - up area may contribute volume scattering. The same reasons are attributed to Low rise - Low dense area with low scattering power in both even and volume scattering powers.

Scattering variations in High Rise and Low Rise and Dense and Sparse built up is attributed to i) shape, ii) size of target and iii) orientation angle of polarization of the SAR data.

4.2.6 LULC Classification: Supervised neural network classification of m-delta decomposition was carried out. Accuracy assessment is carried out by generating random points and reference from Google Earth imageries. Over all accuracy of $81.1 \%$ was observed in the study.

Hybrid Polarimetric data provides a basis for classification based on the structural characteristics of the target.

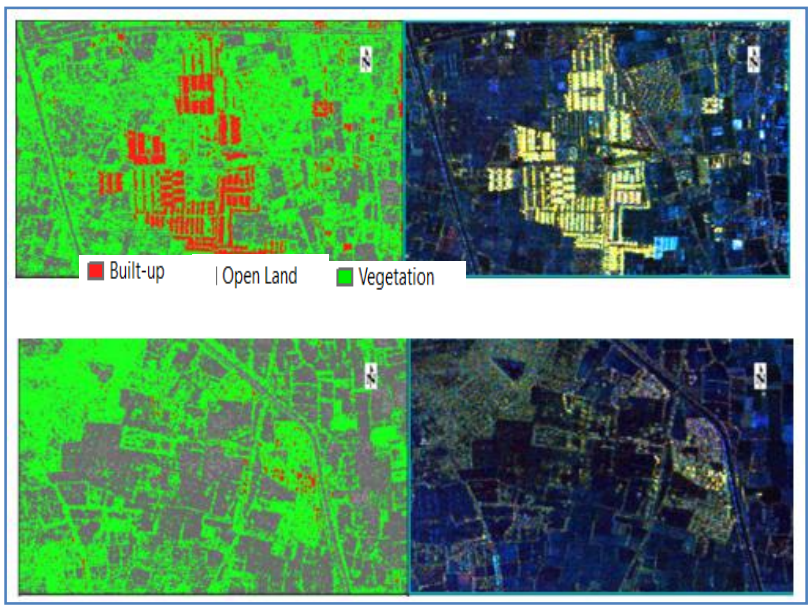

Figure 9: Patches of built-up area in classified image compared with m-delta decomposition

\section{CONCLUSIONS}

The present study addressed the potential of airborne NISAR data to discriminate the land cover classes emphasizing the urban area. This has been carried out by generating polarimetric decompositions using Compact polarimetric data (m-delta, mchi and $\mathrm{m}$-alpha decompositions) in $\mathrm{L}$ wavelength band. $\mathrm{M}$ delta decomposition image showed encouraging result for odd, even and volume scattering of various features. It is also observed that building orientation and density has effect on scattering pattern.

With respect to building orientation, North - South oriented buildings showed relatively high-volume scattering compared to other orientation buildings. Hybrid Polarimetric data provides a basis for classifier based on the structural characteristics of the target and M-Delta NN classifier is carried out for built-up area discrimination.

Though we were not able to classify the oriented and orthogonal built-up area in the present study, it will provide an insight to address it using compact polarimetric data. The analysis carried out viz., variation of scattering power with respect to orientation of the building and density of the buildings will be useful for future studies.

\section{ACKNOWLEDGEMENTS}

This study has been carried out as a part of SAC-CEPT collaborative project "SAR Remote Sensing of Urban area" under Announcement of Opportunity $\mathrm{L}$ and $\mathrm{S}$ band SAR programme of SAC, ISRO.

\section{REFERENCES}

Charbonneau, F. J., Brisco, B., Raney, R. K., McNairn, H., Liu, C., Vachon, P. W., Shang, J., DeAbreu, R., Champagne, Merzouki, A., 2010. Compact Polarimetry Overview and Applications Assessment. Canadian Journal of Remote Sensing, 36 (sup2), 298-315.

Jayasri, P., Niharika, K., Joseph, M., Ryali, H., Sarma, C., Kumari, E., Prasad, A., 2018. Implementation of RISAT-1 
Hybrid Polarimetric Decomposition Techniques and Analysis Using Corner Reflector Data. Journal of the Indian Society of Remote Sensing., 46 (6), 1005-1012 doi.org/10.1007/s12524018-0758-2.

Raney, K., Spudis, P. D., Bussey, B., Crusan, J., Robert Jensen, J., Marinelli, W., Mc Kerracher, P., Neish, C., Palsetia, M., Schulze, R., 2011.The Lunar Mini-Rf Radars: Hybrid Polarimetric Architecture and Initial Results. Proceedings of the IEEE, 99 (5): 808-823.

Raney, R.K., 2006. Dual-polarized SAR and Stokes parameters. IEEE Geosci. Remote Sens. Lett., 3, 317-319.

Raney, R.K., 2007. Hybrid-Polarity SAR Architecture. IEEE Trans. Geosci. Remote Sens., 45 (11), 3397-3404.

Raney, R.K., Cahill, J. T. S., Patterson, G. W., Bussey, D. B. J., 2012. The $\mathrm{m}$-chi decomposition of hybrid dualpolarimetric radar data. IEEE International Geoscience and Remote Sensing Symposium., 5093-5096.

Saran, S., Das, A., Mohan, S., Chakraborty, M., 2012. Study of Scattering Characteristics of Lunar Equitorial Region using Chandrayaan-I Mini-SAR Polarimetric data. Planet. Space Sci., 71 (1), 18-30.

Sivasankar, T., Kumar, D., Srivastava, H. S., Patel, P., 2019. Wheat leaf area index retrieval using RISAT-1 hybrid polarized SAR data. Geocarto Int., 1-11.

Sivasankar, T., Srivastava, H.S., Sharma, P., Kumar, D., Patel, P., 2015. Study of Hybrid Polarimetric Parameters generated from RISAT-1 SAR data for various Land Cover targets. International Journal of Advancement in Remote Sensing, GIS and Geography. 3. 32-42.

Turkar, V., De, S., Ponnurangam, G. G., Deo, R. Rao, Y. S. and Das, A., 2013. Classification of RISAT-1 hybrid polarimetric data for various land features. Proceedings of Asia-Pacific Conference on Synthetic Aperture Radar (APSAR), 494-497.

Uppala, D., Kothapalli, R. V., Poloju, S., Mullapudi, S. S. V. R. and Dadhwal, V. K., 2015. Rice crop discrimination using single date RISAT1 hybrid pol. Data. Photogrammetric Eng. Remote Sens., 81 (7), 557-563.

Xie, L., Zhang, H., Wu, F., Wang, C., and Zhang, B., 2015. Capability of rice mapping using hybrid polarimetric SAR data. IEEE J. Sel. Topics Appl. Earth Observ. Remote Sens., 8 (8), 3812-3822. 